\title{
The Atacama Pathfinder EXperiment (APEX) - a new submillimeter facility for southern skies -
}

\author{
R. Güsten ${ }^{1}$, L. Å. Nyman ${ }^{2}$, P. Schilke ${ }^{1}$, K. Menten ${ }^{1}$, C. Cesarsky ${ }^{2}$, and R. Booth ${ }^{3}$ \\ 1 Max-Planck-Institut für Radioastronomie, Auf dem Hügel 69, 53121 Bonn, Germany \\ e-mail: rguesten@mpifr-bonn.mpg.de \\ 2 European Southern Observatory, Karl-Schwarzschild-Str. 2, 85748 Garching, Germany \\ 3 Onsala Space Observatory, 43992 Onsala, Sweden
}

Received 12 April 2006 / Accepted 19 April 2006

ABSTRACT

\begin{abstract}
APEX, the Atacama Pathfinder EXperiment, has been successfully commissioned and is operational. This novel submillimeter telescope is located at $5107 \mathrm{~m}$ altitude on Llano de Chajnantor in the Chilean High Andes, on what is considered one of the world's outstanding sites for submillimeter astronomy. The primary reflector with $12 \mathrm{~m}$ diameter has been carefully adjusted by means of holography. Its surface smoothness of only 17-18 $\mu \mathrm{m}$ makes APEX suitable for observations up to $200 \mu \mathrm{m}$, through all atmospheric submm windows accessible from the ground. First scientific results will be presented in the accompanying papers of this special issue.
\end{abstract}

Key words. telescopes - submillimeter

\section{Introduction}

The Atacama Pathfinder EXperiment (APEX) is operational. After successful commissioning and science verification the project was inaugurated on 2005 Sep. 25. Located at an altitude of $5107 \mathrm{~m}$ on Llano de Chajnantor in the Chilean High Andes, on what is considered one of the world's outstanding sites for submillimeter astronomy, the APEX opens unmatched scientific opportunities for the study of the submm/FIR wavelength regimes (Fig. 1).

As its name says, APEX is the pathfinder for other submm wavelength missions, most directly for the Atacama Large Millimeter Array (ALMA) - an interferometer array of at least 50 submm telescopes. APEX actually is one of the prototypes modified for the needs of single dish operations. There are great complementarities to the Herschel Satellite (the 4th cornerstone in ESA's "Horizons 2000" program) and the Stratospheric Observatory for Infrared Astronomy (SOFIA), whose instruments will reach to higher frequencies into the infrared wavelength range and will operate in wavelength regimes not accessible from the ground.

\section{The Project - a brief history}

APEX is a collaboration between the Max-Planck-Institut für Radioastronomie (MPIfR), the Onsala Space Observatory (OSO) and the European Southern Observatory (ESO). The observing time (45\% MPIfR, 24\% ESO, 21\% OSO) will be shared along the Partner's investments, with $10 \%$ allocated to the Chilean host nation. The operation of the facility has been entrusted to ESO (Chile).

Shortly after the Partners signed the APEX Memorandum of Understanding on July 02, 2001 MPIfR contracted the design and construction of the telescope to VERTEX Antennentechnik $\mathrm{GmbH}$, Germany. Construction at the high site started in spring
2003. Rolf Güsten has been the Project Manager since spring 2004, during the commissioning phase; with operational readiness the responsibility for operation has been transferred to the Station Manager Lars-Åke Nyman.

\section{The facility}

The telescope is routinely operated by radio link (36 Mbits/s) from the base in Sequitor (altitude $2440 \mathrm{~m}$ ), a few $\mathrm{km}$ south of the village of San Pedro de Atacama. At the base, laboratories and offices as well as dormitories and a cafeteria provide the infrastructure necessary for efficient operation of this remote facility. The staff of 25 people, including astronomers, engineers, technicians and maintenance personnel, is contracted to ESO.

The high site is accessed via the international highway to Paso de Jama and Argentina for the first $60 \mathrm{~km}$, followed by another $15 \mathrm{~km}$ of dirt road to Llano de Chajnantor (ALMA site). The infrastructure at the high site includes an oxygenized control room, laboratories and storage containers. Electrical power is generated by two (redundant) diesel generators $(250 \mathrm{kVA}$ at the high altitude). Work at the high altitude is governed by strict safety rules; access to the site requires authorization by the Station Manager.

\section{The telescope}

The telescope is a modified ALMA prototype, customized to accommodate two Nasmyth cabins for heterodyne receivers and two large instrument containers for supplementary equipment (Fig. 2).

The $12 \mathrm{~m}$ diameter main dish consists of 264 aluminium panels in 8 rings fixed on a carbon fibre reinforced plastic (CFRP) backup structure of 24 sandwich shell segments. Each panel is supported by five vertical (four corners and center) and three 


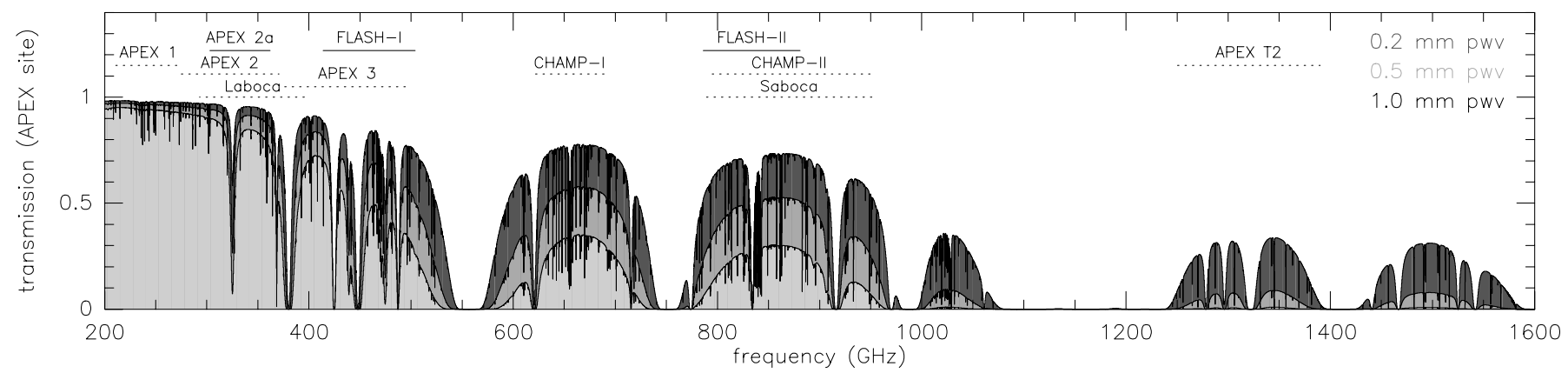

Fig. 1. Zenith transmission of the atmosphere above Llano de Chajnantor at submillimeter wavelengths. Using data from the ALMA site characterization data base covering the years 1995 to 2004 , we calculate that the median column of precipitable water is about $1.2 \mathrm{~mm}$ and the $25 \%$ quartile about $0.7 \mathrm{~mm}$, including data taken during the Bolivian winters. During the winter months the median drops by a factor 2-3. The project plan requires that all atmospheric windows accessible from ground shall be covered by state-of-the-art instruments. We superimpose the frequency coverage of the APEX facility and PI receivers, as they are in operation now (solid lines) and as committed for delivery (dotted). Several contributions to this special issue are dedicated to our instruments.

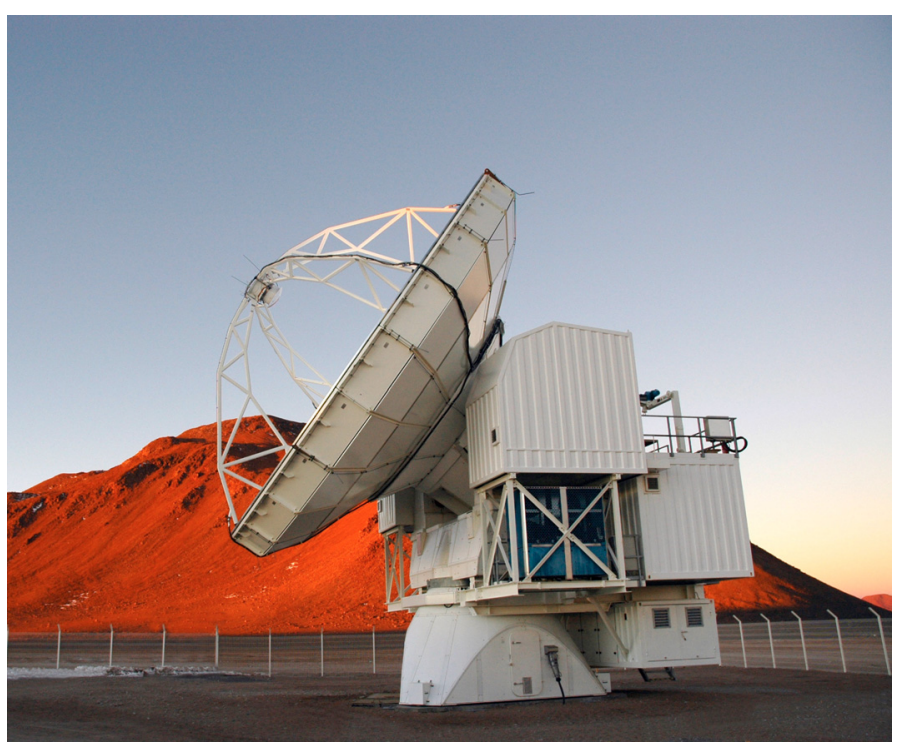

Fig. 2. The APEX telescope at sunset with Cerro Chajnantor in the background (photo kindly provided by A. Lundgren, APEX).

horizontal adjustment elements. The panels, which have been chemically etched to scatter visible and IR radiation and thus allow daytime observations, have been manufactured to an average surface accuracy of $8 \mu \mathrm{m} \mathrm{rms}$. The backup structure (BUS) is supported by an INVAR cone, which is attached to the top of the Cassegrain cabin. The total mass of the modified antenna is $\sim 125$ tons.

The optical parameters of the telescope are summarized in Table 1. The telescope is a Cassegrain system with a paraboloidal main reflector, on an elevation over azimuth mount. The aluminium secondary reflector (surface roughness better than $4 \mu \mathrm{m}$ ) is supported by CFRP quadripod legs, position control is provided by a high-precision hexapod. The sub-reflector does allow for wide-field bolometer arrays in the Cassegrain cabin. For operation (of basically the heterodyne receivers) in the Nasmyth cabins, the telescope waist from the secondary is transformed by refocusing optics (through the elevation tube with minimum free diameter of $150 \mathrm{~mm}$ only) into the Nasmyth waists. As refocusing system we use a Gaussian mirror telescope with frequency independent optics; the tertiary mirror package is on a rotary support to select between the two Nasmyth foci and to clear the
Table 1. Optical design parameters.

\begin{tabular}{ll}
\hline \hline Paraboloidal main reflector & \\
diameter [m] & $D=12.0$ \\
focal length [m] & $f=4.8$ \\
focal ratio & $f / D=0.40$ \\
illumination angle [deg] & $\theta_{\mathrm{p}}=128.0$ \\
Hyperboloidal secondary reflector & $d=0.75$ \\
diameter [m] & $2 c=6.177$ \\
distance primary - secondary focus [m] & $a=0.75$ \\
distance focus - elevation axis [m] & $L=-0.294$ \\
distance primary focus - secondary [m] & $\theta_{\mathrm{s}}=7.2$ \\
illumination angle [deg] & $M=20$ \\
magnification factor & $f / D=8.0$ \\
final focal length &
\end{tabular}

optical path to the bolometer pick-up mirror (on the floor of the Cassegrain cabin).

\section{Verification of design goals}

The APEX specifications require a surface accuracy of better than $20 \mu \mathrm{m}$ rms, a tracking accuracy of $0.6^{\prime \prime}$ (within 2 degrees to calibrator) and an absolute pointing accuracy of $2^{\prime \prime} \mathrm{rms}$ for primary observing conditions ${ }^{1}$.

The tracking and pointing performance was verified by optical pointing on stars to comply with these requirements. Our radio pointing is based on this optical pointing model. Only those terms were determined by submillimeter measurements that are in addition required for operation from the submillimeter foci of the antenna. Despite the scarceness of bright submm sources (there is no bolometer in operation so far), typically an all-sky rms of $2-3^{\prime \prime}$ can be expected during stable night time conditions.

\subsection{Holographic adjustment of the surface}

After assembly, the main reflector was adjusted by means of optical photogrammetry (by VERTEX) to $35-40 \mu \mathrm{m} \mathrm{rms}$ surface accuracy. From there on the APEX holography team performed near-field holography with a $92.4 \mathrm{GHz}$ transmitter (phase-locked, output power $10 \mathrm{~mW}$ ) located near the summit of

\footnotetext{
1 Primary conditions are defined by ambient temperatures from -20 to $+20{ }^{\circ} \mathrm{C}$ and for average wind loads of not more than $9 \mathrm{~m} / \mathrm{s}$ (night) and $6 \mathrm{~m} / \mathrm{s}$ (daytime).
} 
Cerro Chajnantor, at an elevation of $\sim 13 \mathrm{deg}$. The main receiver was located in the telescope waist position (inside the Cassegrain cabin), while the reference receiver was mounted behind the subreflector, facing the transmitter - this way the combined optical system of primary and secondary reflector is adjusted (details of the experimental setup and data analysis will be presented separately, Sridharan et al. 2006).

Data were taken while fast ( $1050^{\prime \prime} / \mathrm{s}$ slews, $2 \mathrm{~ms}$ dumps) onthe-fly scanning $1.5 \times 1.5$ degree-sized fields centred towards the transmitter (which translates into aperture power and phase maps of $128 \times 128$ pixels, corresponding to $\sim 10 \mathrm{~cm}$ spatial resolution on the aperture). A vector voltmeter served as backend to record the complex data. Data processing was performed in the framework of the SMA data analysis package (Sridharan et al. 2004; Zhang 1997), adapted to the needs of APEX. The phase residuals were converted to surface error maps which were used to correct for panel-to-panel misalignments and panel flexure. Comparison of subsequent holography maps showed an excellent short-term repeatability of the measurements of $5.5 \mu \mathrm{m}$ rms only.

Holography was performed in three sessions (May and June 2004, April 2005). Typically, the manual adjustment of the (maximally) 1320 vertical adjuster elements (INVAR) took a full workday, depending on the environmental conditions, with rotating teams. In April 05, then also allowing for non-planar surface profiles of the panels, the surface was set and verified to excellent $15 \mu \mathrm{m}$ rms accuracy (Fig. 3), which is well below specifications.

Dependent on the solar illumination angle and temperature gradients introduced, the surface will degrade by a few $\mu \mathrm{m}$ during daytime and in particular during sunrise/setting. We found no evidence for a longer-term degradation of the antenna setting since the first adjustment in summer 2004. Our tests clearly revealed, that in order to maintain this outstandingly good performance, the temperature distribution of the BUS support has to be stable (which is achieved by active temperature stabilization of the Cassegrain cabin, and hence the INVAR cone, to $13 \pm 1{ }^{\circ} \mathrm{C}$ ).

With the antenna thus optimized at 13 deg elevation, gravitational deformation would rapidly degrade the performance towards higher elevations. We used the finite element model of the antenna to pre-load the surface settings for an elevation of $50 \mathrm{deg}$ - thus optimizing the performance for the elevation range that will actually be used for astronomical observations. The effective surface accuracy is calculated to be $\sim 17-18 \mu \mathrm{m}$ for the elevation range $30-80 \mathrm{deg}$.

\subsection{Telescope efficiencies measured on sky}

An independent way to characterize the sensitivity of a reflector antenna is by astronomical determination of the coupling efficiencies by observing planets (e.g., Baars 1973; Kramer 1997; Downes 1989). The basic parameter, the aperture efficiency $\eta_{\mathrm{a}}(\lambda)$, the ratio between the effective and the geometric area of the antenna is, however, difficult to measure, requiring full control about the absolute calibration of the complete system.

The effective aperture efficiency is determined by a number of contributions, several of which are rather difficult to quantify. For the purpose of our discussion we separate

$\eta_{\mathrm{a}}(\lambda)=A_{\mathrm{e}} / A_{\mathrm{g}}=\eta_{0} \eta_{\mathrm{R}}(\lambda)=\eta_{0} \eta_{\mathrm{R}, 0} \exp \left(-(4 \pi \epsilon / \lambda)^{2}\right)$.

$\eta_{\mathrm{R}}(\lambda)$ describes the frequency dependent surface error efficiency (Ruze losses due to small random surface errors, Ruze 1966). Fitting $\eta_{\mathrm{R}}(\lambda)$ does in principle allow to determine the surface accuracy $\epsilon . \eta_{0}$ includes all the other losses (like the illumination losses due to the taper of the feed $\eta_{\mathrm{i}}$ (receiver dependent); Ohmic

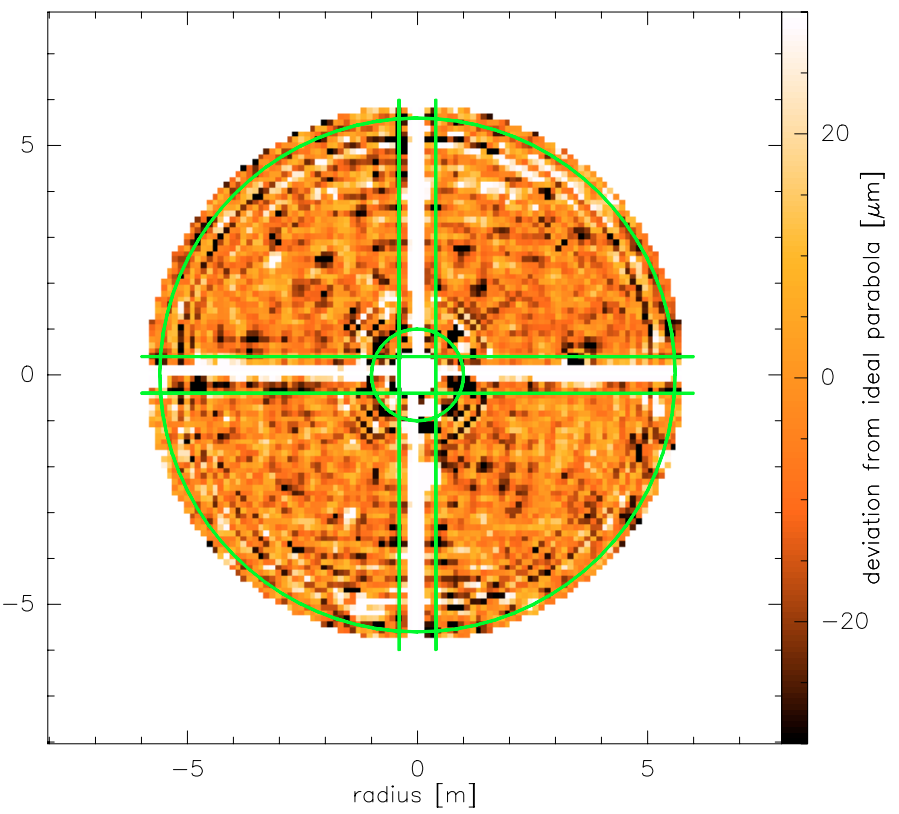

Fig. 3. The surface error pattern of the APEX main reflector after the April 2005 holography session. Masking the inner $1 \mathrm{~m}$ and the projected area of the quadrupod legs, but without radial tapering, the surface accuracy is determined to be $14.7 \mu \mathrm{m} \mathrm{rms}$.

losses with the metal reflector $\eta_{\mathrm{r}}$; blockage by the sub-reflector and its supports $\eta_{\mathrm{b}}$; etc. - see Baars (2003) for a breakdown of the error budgets).

The actual astronomical measurement determining the aperture efficiency $\eta_{\mathrm{a}}(\lambda)$ is rather straightforward: the antenna temperature measured towards a given planet, $T_{\mathrm{a}}^{*}$ (corrected for atmospheric losses and telescope spillover $\eta_{\mathrm{f}}$ ), as provided by the APEX calibration pipeline, is compared to the expected flux density per beam, $S_{v, \mathrm{~b}}[\mathrm{Jy}]$

$\eta_{\mathrm{a}}=2 k\left(T_{\mathrm{a}}^{*} \eta_{\mathrm{f}}\right) /\left(A_{\mathrm{g}} S_{\nu, \mathrm{b}}\right)=24.4\left(T_{\mathrm{a}}^{*} \eta_{\mathrm{f}}\right) / S_{\nu, \mathrm{b}}$

where $k$ is the Boltzmann constant. Similarly, the main beam efficiency is derived from measurements of planets (of size $\theta_{\mathrm{s}}$, and brightness temperature $T_{\mathrm{b}}$ )

$\eta_{\mathrm{mb}}=\left(T_{\mathrm{a}}^{*} \eta_{f}\right) / J_{v}\left(T_{\mathrm{b}}\right)\left[1-\exp \left(-\ln (2)\left(\theta_{\mathrm{s}} / \theta_{\mathrm{b}}\right)^{2}\right)\right]$

Results of the efficiency measurements for the APEX receivers currently in operation are compiled in Table 2 . Ruze losses $\eta_{\mathrm{R}}(\lambda)$ are displayed in Fig. 4 (see legend of figure for details). The data are nicely consistent with the surface accuracy predicted from our holography: using the high-frequency data (from FLASH \& CONDOR) only the data are consistent with $17 \mu \mathrm{m} \mathrm{rms} \mathrm{accu-}$ racy, including the (more uncertain) APEX-2a measurements we derive $18 \mu \mathrm{m}$, and ignoring the CONDOR point, an upper limit of $20 \mu \mathrm{m}$ can be set.

In order to maintain these good coupling efficiencies, the sub-reflector must be tracked with high precision (by moving the hexapod) at close-to-nominal foci positions under gravity and thermal loads. We confirmed the variation of focus positions with elevation as calculated by VERTEX from their Finite Element model of the APEX, but additional temperature dependent corrections (temperature of the aluminium ring supporting the subreflector) had to be added to the hexapod position control. The resulting telescope "gain curve" (for stable night time conditions) is - as desired - nicely elevation independent (Fig. 5), which also supports the validity of the APEX calibration pipeline. 
Table 2. Telescope efficiencies.

\begin{tabular}{ccccccl}
\hline \hline$v[\mathrm{GHz}]$ & $\theta_{\mathrm{mb}}\left[{ }^{\prime \prime}\right]$ & $\eta_{\mathrm{f}}$ & $\eta_{\mathrm{a}}$ & $\eta_{\mathrm{mb}}$ & $\eta_{\mathrm{M}}$ & Receiver \\
\hline 352 & 17.3 & 0.97 & 0.60 & 0.73 & 0.85 & APEX-2a \\
464 & 13.3 & 0.95 & 0.51 & 0.60 & 0.81 & FLASH-I \\
812 & 7.7 & 0.95 & 0.35 & 0.43 & 0.79 & FLASH-II \\
\hline
\end{tabular}

Notes: main beam widths have been determined from de-convolved continuum slews across small planets (Mars, Uranus). The forward efficiency derives from total power sky-dips. Aperture and main-beam efficiencies $\eta_{\mathrm{a}}, \eta_{\mathrm{mb}}$ are averages from typically 5-10 individual measurements of Mars (in June 2005 Mars was compact, $\theta_{\mathrm{s}} \sim 8^{\prime \prime}$ ). Moon efficiencies have been determined for full moon (equivalent BB temperatures from Pardo et al. 2005). Body temperatures and sizes for the planets are taken from the fluxes program provided by the JCMT (http://www.jach.hawaii.edu/jac-bin/planetflux.pl). Efficiencies should be accurate to 5-10\%, with quite some uncertainties about the actual body temperature of the planets.

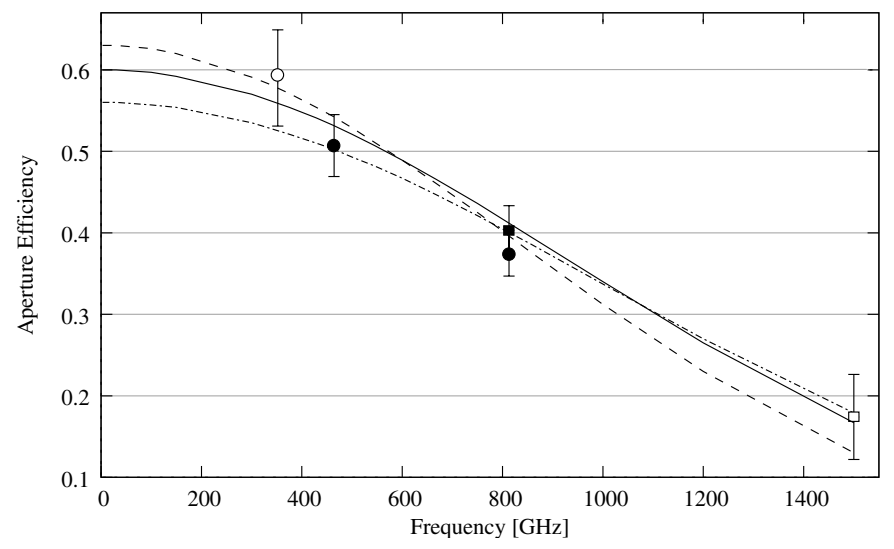

Fig. 4. Aperture efficiency $\eta_{\mathrm{R}}$ (Ruze losses) versus frequency. Filled symbols denote measurements with FLASH (Heyminck et al. 2006) of Mars (circles) and Jupiter (squares); open symbols are observations with the APEX-2a receiver (Risacher et al. 2006; error bars raised to $10 \%$ because of a compression problem in the continuum detector). Data taken with the CONDOR receiver (Wiedner et al. 2006) have been re-processed. $\eta_{\mathrm{R}}$ has been derived from the aperture efficiencies $\eta_{\mathrm{a}}$ (Table 2), after correction for differential illumination losses $\eta_{\mathrm{i}}$, Ohmic and Ruze losses on the tertiary mirrors inside the Cassegrain cabin, and transmission losses in the Gore-Tex foil closing the Cassegrain cabin (for details, see Güsten et al. 2006).

Properly focussed, the APEX beam pattern looks clean as designed: the beam width determined by continuum slews across the smaller planets is fully consistent with diffraction limited optics $\left(\theta_{\mathrm{mb}}=1.2 \lambda / \mathrm{D}\right.$, Table 2$)$, and the error pattern is of low amplitude. From analysis of $464 \mathrm{GHz}$ total power scans across the Moon (following the method described in Greve et al. 1998) we derive the first error beam at $-30 \mathrm{~dB}$ from the main beam, consistent with our holography results. The width of the error beam corresponds to the size of the individual panels $\left(\theta_{\text {err }} \sim 180^{\prime \prime}\right.$ at $464 \mathrm{GHz})$.

\section{The next steps}

The next major milestone will be the integration of the chopping secondary, to be followed by a holography with the then final optical configuration of the telescope. Major instruments are expected for commissioning this year: the large bolometer camera LABOCA (facility status), the dual-colour $\mathrm{CHAMP}^{+}$heterodyne array (principal investigator MPIfR,

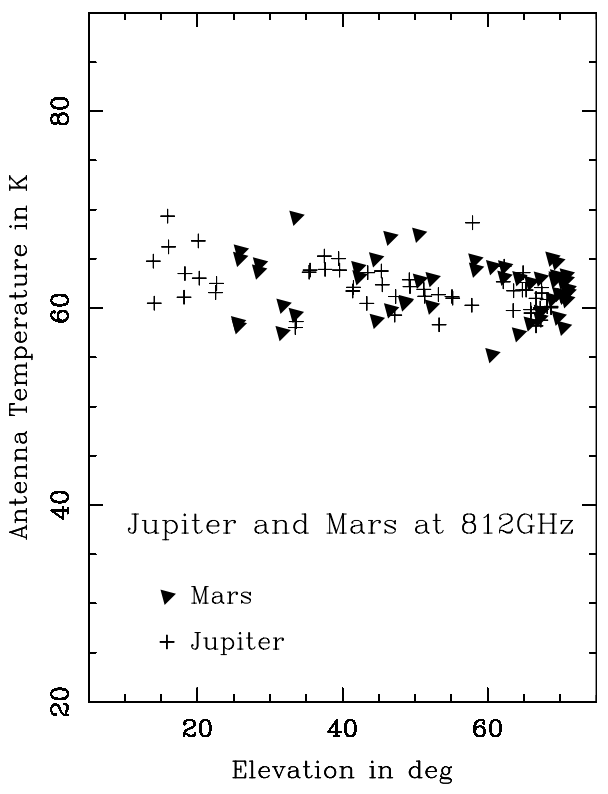

Fig. 5. "Gain curve" of the APEX, displaying the antenna temperature of Jupiter and Mars vs. elevation as provided by the online calibrator. Temperatures for Mars have been scaled.

Kasemann et al. 2006), and later this year, the facility heterodyne receiver with mixers up to $500 \mathrm{GHz}$ (delivery from OSO).

Acknowledgements. The realization of the APEX, on an amazingly compressed schedule, has only been possible due to the enthusiasm and outstanding support by our very committed teams, all sharing the desire to make this outstanding facility become reality. We acknowledge the excellent collaboration with the VERTEX Project Management and constructor teams - the determination of W. Häupl during the telescope construction under harsh winter mountain conditions kept us on schedule.

We like to emphasize the support we received from the holography project team, consisting of T. K. Sridharan and N. Patel from the Harvard-Smithsonian Center for Astrophysics (CfA), and A. Greve and D. Morris (both retired from IRAM). The holography equipment has been loaned from the SMA, which is acknowledged with pleasure.

We thank the APEX staff and our teams at the home institutes, whose outstanding support has been so essential during the build-up and commissioning phase. Times have not been easy during those months, but at the end of the days we delivered a world-leading facility that already during its first few months of operation has demonstrated its great potential for outstanding science.

\section{References}

Baars, J. W. M. 1973, IEEE, 21, 461

Baars, J. W. M. 2003, Alma Memo, 456

Downes, D. 1989, in Diffraction-Limited Imaging with Very Large Telescopes, ed. D. M. Alloin \& J.-M. Mariotti, NATO ASIC Proc., 274, 53

Greve, A., Kramer, C., \& Wild, W. 1998, A\&AS, 133, 271

Güsten, R., Nyman, L.-Å., Menten, K. M., et al. 2006, in Ground-based and Airborne Telescope, Proc. SPIE, 6267, in preparation

Heyminck, S., Kasemann, C., Güsten, R., de Lange, G., \& Graf, U. U. 2006, A\&A, 454, L21

Kasemann, C., Güsten, R., Heyminck, S., et al. 2006, in Millimeter and Submillimeter Detectors and Instrumentation for Astronomy III, Proc. SPIE, 6275 , in preparation

Kramer, C. 1997, IRAM Internal Technical Memo

Pardo, J. R., Serabyn, E., \& Wiedner, M. C. 2005, Icarus, 178, 19

Risacher, C., Vassilev, V., Monje, R., et al. 2006, A\&A, 454, L17

Ruze, J. 1966, Proc. IEEE, 54, 633

Sridharan, T. K., Greve, A., Morris, D., et al. 2006, A\&A, in preparation

Sridharan, T. K., Saito, M., Patel, N. A., \& Christensen, R. D. 2004, in Ground-

based Instrumentation for Astronomy, ed. A. F. M., Moorwood, M. Iye,

J. Antebi \& D. Lemke, Proc. SPIE, 5495, 441

Wiedner, M. C., Schmidt, G., Bielau, F., \& Rettenbacher, K. 2006, A\&A, 454, L33

Zhang, X. 1997, SMA Technical Memo, 116 